\title{
Effect of soil moisture content on corrosion behavior of X70 steel
}

Fei Qin ${ }^{1}$, Changliang Jiang ${ }^{2}$, Xinhua Cui ${ }^{2}$, Qiang Wang ${ }^{2}$, Juhong Wang ${ }^{2}$ Rui Huang ${ }^{2}$, Datao Yu, Qing Qu ${ }^{3}$, Yingjie Zhang ${ }^{1,4}$, Peng Dong Peng ${ }^{1,4, *}$

${ }^{1}$ National and Local Joint Engineering Laboratory for Lithium-ion Batteries and Materials Preparation Technology, Key Laboratory of Advanced Battery Materials of Yunnan Province, Faculty of Materials Science and Engineering, Kunming University of Science and Technology, Kunming 650093, China.

${ }^{2}$ CNPC South-east Asia Pipeline Co.,Ltd, Beijing 100000,China

${ }^{3}$ Faculty of chemical science and Engineering, Yunnan university, Kunming 650091, China

${ }^{4}$ Faculty of Metallurgical and Energy Engineering, Kunming University of Science and Technology, Kunming 650093, China.

*E-mail: dongpeng2001@ 126.com

doi: $10.20964 / 2018.02 .32$

Received: 23 September 2017 / Accepted: 23 November 2017 / Published: 28 December 2017

The effects of moisture content on corrosion of X70 steel in soils from different regions containing Muse, Tungtha, Made Kyun and Made Kyun beach in Myanmar were studied by polarization curves, electrochemical impedance spectroscopy and SEM. It was found that the corrosion rate law of X70 steel in each studied soil tends to be different with the change of moisture content due to the combined effects between soil moisture content and chloride ion content. The values of the corrosion rate were maxima with soil moisture content of $20 \%$ except that for the Muse soil where the corrosion rate was minimal with moisture content of $20 \%$. The values of $\mathrm{E}_{\text {corr }}$ were shifted to more negative values with increasing soil moisture content of up to $60 \%$, and then there was an appreciable increase in the value of $E_{\text {corr }}$. However, the value of $E_{\text {corr }}$ was shifted to more negative value with increasing Made Kyun soil moisture content of up to $80 \%$. A good uniformity between the data obtained from polarization curves and EIS measurements results. X70 steel was serious corroded in Made Kyun beach and Made Kyun regions via the data of EIS, polarization curves and SEM result from the different content of chloride in different areas.

Keywords: X70 steel; soil corrosion; moisture content; EIS; polarization curve 
(C) 2018 The Authors. Published by ESG (www.electrochemsci.org). This article is an open access article distributed under the terms and conditions of the Creative Commons Attribution license (http://creativecommons.org/licenses/by/4.0/). 